\title{
ON THE INVERSE IMAGE OF BAIRE SPACES
}

\author{
MUSTAFA ÇIÇEK \\ Department of Mathematics \\ Faculty of Science \\ Ankara University \\ 06100 Beşevler Ankara - TÜRKIYE
}

(Received September 18, 1995 and in revised form March 12, 1996)

\begin{abstract}
In 1961, Z. Frolik proved that if $f$ is an open and continuous mapping of a metrizable separable space $X$ onto Baire space $Y$ and if the point inverses are Baire spaces, then $X$ is a Baire space. We give a generalization to semi-continuous and semi-open mapping of this theorem and extended it to the several types of mappings.
\end{abstract}

KEY WORDS AND PHRASES: Baire spaces, second category spaces, second countable space, metrizable separable space, almost-regular spaces, nowhere dense set, weakly-continuous, semi-continuous, almost-continuous, feebly-continuous, almost-open mapping, semi-open mapping, feebly-open mapping, weakly-open mapping.

1991 AMS SUBJECT CLASIFICATION CODES: Primary 54E52, 54C08, 54C10 secondary 54D10, 54D65, 54E35.

\section{O. INTRODUCTION}

The inverse image of a Baire space does not need to be a Baire space, even in the case when the mapping is a continuous open surjection for which each point inverse is a Baire space. Indeed, In [18], Oxtoby gave an example of a Baire space $X$ such that the cartesian product $X \times X$ is not a Baire space, but the projection mapping from $X \times X$ onto $X$ is continuous open for which each point inverse is a Baire space. Later in [13], Krom showed that if there is such an example then there is also a metric Baire space whose square is not a Baire. In fact, he used the example given by Oxtoby in [18] to answer, negatively, the open question of whether products of metric Baire spaces are Baire [5, p120], [18, p159] and [20, p292] and showed how to obtain from any Baire space $X$, an associated metric Baire space $K$ (a space of countable sequences with the Baire metric [23, p124]) such that $X \times X$ is a Baire space iff $K \times K$ is a Baire space, where the space $K$ is an ultrametric space $[6, \mathrm{p} 38]$.

The concept of $\theta$-continuity was introduced by S.V.Fomin [8]. In $1961 \mathrm{~N}$. Levine [14] introduced 
weak-continuity and in 1963 he [15] defined semi-continuity as a weakened form of continuity. On the other hand, in 1968 , M.S.Singal and A.R.Singal [21] introduced almost-continuous mappings, almost-open mappings Moreover, M.Biswas [2] defined semi-open mappings which contain the class of open mappings and in 1984 D.A.Rose [19] introduced weakly-open mappings as a new weakened form of open mappings.

This paper studies the inverse image of second category spaces and Baire spaces under these various types of mappings. Here we consider the following mappings $f: X \rightarrow Y$ which are;

(i) $f$ is semi-open and semi-continuous

(ii) $f$ is semi-open and almost-continuous

(iii) $f$ is feebly-open and almost-continuous

(iv) $f$ is weakly-open and weakly-continuous

(v) $f$ is weakly-open and $\theta$-continuous

(vi) $f$ is weakly-open and almost-continuous

(vii) $f$ is almost-open and almost-continuous

In section 2 at first a basic theorem (Theorem 2.5) which we need the proof of our main theorems is given as a generalization to the above first three classes of mappings of [9, Theorem $2^{\prime}$ ] on the inverse image of second category spaces. Here the space $X$ is assumed to be second countable. Furthermore we show that this theorem can be extended to the classes of mappings which are given in the above last four cases, in the case when $X$ is a metrizable separable space (Corollary 2.6) and it is also valid for feebly-open and weakly-continuous ( $\theta$-continuous) mappings in the case when $X$ is second countable space and $Y$ is almost-regular space (Corollary 2.7). And then we give the proof of our main theorems concerning the inverse image of Baire spaces. The first of them (Theorem 2.1) is a generalization to semi-open and semi-continuous mapping of [9, Theorem 2] in the case when $X$ is a second countable space. Second one (Theorem 2.2) is an extention to the classes of mappings which are given in the above last four cases of $[9$, Theorem 2 ] in the case when $X$ metrizable separable space.

The most frequently used notations follow. Let $A$ be a subset of a topological space $X$. The closure of $A$ in $X$ and interior of $A$ in $X$ will be denoted by $\bar{A}$ and $\operatorname{int} A$ respectively. The complement of $A$ in $X$ is $X-A$. Throughout this paper $X$ and $Y$ will always denote topological spaces on which no separations axiom are assumed unless stated explicitly. No mapping is assumed to be continuous unless stated.

\section{PRELIMINARIES}

In this section we give a survey of notions and background results used in this paper.

DEFINITION 1.1. Let $A$ be a subset of $X . A$ is said to be a semi-open [15] if there exists an open subset $O$ of $X$ such that $O \subset A \subset \bar{O}$.

The following results can be found in [15].

THEOREM 1.2. A subset $A$ in $X$ is semi-open if and only if $A \subset \overline{\operatorname{int} A}$. 
THEOREM 1.3. Let $A \subset Y \subset X$. If $Y$ is a subspace of $X$ and $A$ is semi-open subset in $X$, then $A$ is semi-open subset in $Y$.

THEOREM 1.4. If $A$ is open and $S$ is a semi-open subset of $X$, then $A \cap S$ is semi-open in $X[7$, Theorem 1.9].

DEFINITION 1.5. A mapping $f: X \longrightarrow Y$ is said to be semi-continuous [15] (resp., semiopen [2]) if for each open subset $V$ in $Y$ (resp. open subset $U$ in $X), f^{-1}(V)$ is semi-open in $X$ (resp. $f(U)$ is semi-open in $Y$ ).

The following results which will be needed for our proof in the sequel.

THEOREM 1.6. Let $f: X \longrightarrow Y$ be semi-open and let $A$ be an open subset of $X$. Then $f_{/ A}: A \longrightarrow f(A)$ defined by $f_{/ A}(x)=f(x)$, for all $x \in A$ is semi-open .

PROOF. Let $U$ be any open set in $A$. Since $A$ is open in $X$, then $U$ is also open in $X$. Since $f$ is semi-open then $f(U)$ is semi-open in $Y$. Consequently $f(U)$ is semi-open in $f(A)$ by Theorem 1.3 .

THEOREM 1.7. Let $f: X \longrightarrow Y$ be semi-continuous and let $A$ be an open subset of $X$. Then $f_{/ A}: A \longrightarrow f(A)$ defined by $f_{/ A}(x)=f(x)$, for all $x \in A$ is semi-continuous.

PROOF. Let $G$ be any open set in $f(A)$. Then there exits an open subset $V$ in $Y$ such that $G=f(A) \cap V$. Consequently $f_{/ A}^{-1}(G)=A \cap f^{-1}(G)=A \cap f^{-1}(f(A) \cap V)$. From this we have $f_{/ A}^{-1}(G)=A \cap f^{-1}(V)$. Since $f$ is semi-continuous, then $f^{-1}(V)$ is semi-open in $X$ and also $A \cap f^{-1}(V)$ is semi-open in $X$ by Theorem 1.4. Hence $f_{/ A}^{-1}(G)=A \cap f^{-1}(V)$ is semi-open in $A$ by theorem 1.3.

DEFINITION 1.8. A mapping $f: X \longrightarrow Y$ is said to be almost-continuous [21] (resp. weakly-continuous[14], $\theta$-continuous [8]) if for each $x \in X$ and each neighborhood $\mathrm{V}$ of $f(x)$, there exists an open neighborhood $U$ of $x$ such that $f(U) \subset \operatorname{int}(\bar{V})($ resp. $f(U) \subset \bar{V}, f(\bar{U}) \subset \bar{V})$.

REMARK 1.9. The following implications are known [17, Remark 1] and neither implication is reversible.

almost continuity $\rightarrow \theta$-continuity $\rightarrow$ weak-continuity

The counter examples to the converse of the first implication and the second implication can be found in [12] and [21] respectively.

DEFINITION 1.10. A topological space $X$ is said to be almost-regular [22] if for each $x \in X$ and each regular open set $\mathrm{G}$ containing $\mathrm{x}$, there exists a regular open set $\mathrm{V}$ such that $x \in V \subset \bar{V} \subset G$.

The following results which give the sufficient conditions for a weakly-continuous mapping to be almost-continuous, will be used in the sequel.

THEOREM 1.11. If $Y$ is almost-regular and $f: X \longrightarrow Y$ is weakly-continuous, then $f$ is almost-continuous [17, Theorem 1.].

THEOREM 1.12. If $f: X \longrightarrow Y$ is a weakly-continuous open mapping, then $\mathrm{f}$ is almostcontinuous [21, Theorem 2.3]. 
We shall require the following known results.

THEOREM 1.13. Let $f: X \longrightarrow Y$ be almost-continuous. Let $A \subset X$ be such that $f(A)$ is open in $Y$. Then $f_{/ A}: A \longrightarrow f(A)$ is almost-continuous [16, Theorem 6].

THEOREM 1.14. A mapping $f: X \rightarrow Y$ is almost-continuous if and only if the inverse image $f^{-1}(B)$ of each regular open set $\mathrm{B}$ in $\mathrm{Y}$ is open in $\mathrm{X}$ [21, Theorem 2.2].

DEFINITION 1.15. A mapping $f: X \rightarrow Y$ is called feebly-continuous [9] (resp. feeblyopen [9]) if for every nonempty open set $\mathrm{V}$ in $\mathrm{Y}$. (resp. for every nonempty open set $\mathrm{U}$ in $\mathrm{X}$ ) $f^{-1}(V) \neq \emptyset$ imlies int $f^{-1}(V) \neq \emptyset$ (resp. $\left.\operatorname{int}(f(U)) \neq \emptyset\right)$.

REMARK 1.16. The following implications hold:

i) semi-open $\longrightarrow$ feebly-open

ii) If the mapping is surjective then semi-continuous $\longrightarrow$ feebly-continuous

These are immediate consequence of their definitions. But the following example, due to $\mathrm{T}$. Neubrunn [24], shows that none of these implications is reversible.

EXAMPLE 1.17. Let $X$ and $Y$ be the set of real numbers with the usual topology. Let the mapping $f: X \longrightarrow Y$ be defined as follows $f(x)=x$, if $x \neq 0$ and $x \neq 1 ; f(0)=1, f(1)=0$. Then $f$ is one-to-one, feebly-continuous and feebly-open but is neither semi-continuous nor semi-open .

However we have the following result which gives some sufficient conditions for a feebly-open mapping to be semi-open .

THEOREM 1.18. Let $f$ be a mapping from $X$ into $Y$. If any one of the following conditions holds, then $f$ is semi-open .

(i) $f$ is feebly-open and weakly-continuous

(ii) $\mathrm{f}$ is feebly-open and $\theta$-continuous

(iii) $f$ is feebly-open and almost-continuous

PROOF. Suppose that (i) holds and let $A$ be any open subset in $X$. To prove that $f$ is semiopen, it is enough to show that $f(A) \subset \overline{\operatorname{int} f(A)}$ by Theorem 1.2 and Definition 1.5. Let $y$ be any point of $f(A)$. Hence there exists a point $\mathrm{x}$ of A such that $y=f(x)$. Let $\mathrm{V}$ be any open neighborhood of $\mathrm{y}$. Since $\mathrm{f}$ is weakly-continuous, there exists an open subset $\mathrm{U}$ containing $\mathrm{x}$ such that $f(U) \subset \bar{V}$. Therefore $x \in(U \cap A)$ and since $\mathrm{f}$ is feebly-open, then $\operatorname{\imath nt}(f(U \cap A)) \neq \emptyset$. Let us put $W=\imath n t(f(U \cap A))$. From this we have $W \subset f(U) \cap \imath n t(f(A))$. Since $f(U) \subset \bar{V}$, we obtain $W \subset \bar{V} \cap \operatorname{\imath nt}(f(A))$. This shows that $V \cap \operatorname{int}(f(A)) \neq \emptyset$. Consequently $y \in \overline{\operatorname{int}(f(A))}$.

It is clear that both the case (ii) and the case (iii) implies the case (i) by the Remark 1.9. Therefore, for both cases we have also that $\mathrm{f}$ is semi-open .

DEFINITION 1.19. A mapping $f: X \rightarrow Y$ is called almost open [21] (resp. weakly-open [19]) if the image of every regular open subset of $X$ is an open subset of $Y$ (resp. for every open set $\mathrm{U}$ of $\mathrm{X}, f(U) \subset \operatorname{int} f(\bar{U}))$.

REMARK 1.20. The following implications are well known [19] and none of these implications is reversible in general, as is shown by the following examples 
openness $\rightarrow$ almost openness $\rightarrow$ weak-openness

EXAMPLE 1.21. Let $X$ be the set of real numbers and $\mathcal{U}$ be the usual topology on $X$. Let the topolgy $\mathcal{T}$ on $X$ be generated by $\mathcal{U} \cup\{U \cap Q / U \in \mathcal{U}\}$, where $Q$ is the set of rational numbers. Then the identity mapping $i:(X, \mathcal{T}) \rightarrow(X, \mathcal{U})$ is almost-open, but it is not open.

EXAMPLE 1.22. Let $X=\{a, b, c, d\}, \mathcal{T}=\{X, \emptyset,\{b\},\{d\},\{b, d\},\{b, c, d\}\}$ and $Y=$ $\{1,2,3\}, \mathcal{T}^{\prime}=\{Y, \phi,\{1\},\{1,2\},\{1,3\}\}$. Let $f:(X, \mathcal{T}) \longrightarrow\left(Y, \mathcal{T}^{\prime}\right)$ be given by $f(a)=1, f(b)=$ $2, f(c)=f(d)=3$. Then $f$ is weakly-open, but since $\{b\}$ is regular open in $(X, \mathcal{T})$ and $f(\{b\})=\{2\}$ is not open in $\left(Y, \mathcal{T}^{\prime}\right)$, then $f$ is not almost-open

We recall the following results which will be used in the sequel

THEOREM 1.23. If $X$ is regular and $f: X \rightarrow Y$ is weakly-open, then $\mathrm{f}$ is open [19, Theorem 7].

THEOREM 1.24. Let $A$ be a subset of $X$. Then, the following properties are equivalent [3, Theorem 1]

(i) $A$ is semi-closed.

(ii) int $\bar{A} \subset A$

(iii) $X-A$ is semi-open .

The following well-known results concerning the Baire spaces which will be used in the sequel, can be found in $[1],[4],[10],[11]$

THEOREM 1.25. Every open subspace of a Baire space is a Baire space.

THEOREM 1.26. Every space which contains a dense Baire subspace is a Baire space.

THEOREM 1.27. Every open subspace of the second category of a space $X$ is a second category subset relative to $X$.

We shall require the following remark in the sequel.

REMARK 1.28. Let $Y$ and $Z$ be subspaces of a space $X$. Suppose $A \subset Y \cap Z$, then the topologies on $A$ induced by those of $Y$ and $Z$ are identical.

\section{INVERSE IMAGES OF BAIRE SPACES}

In this section, at first we give a generalization of Frolik's Theorem [9, Theorem 2,p.383] on the inverse image of Baire spaces which was cited in the abstract of this paper. And also we extend it to several types of mappings.

THEOREM 2.1. Let $\mathrm{f}$ be a semi-open and semi-continuous mapping from a second countable space $X$ on to a Baire space $Y$. If the sets of the form $f^{-1}(y), y \in Y$ are Baire spaces, then $X$ is a Baire space.

THEOREM 2.2. Let $f$ be a mapping of metrizable separable space $X$ onto a Baire space $Y$. Suppose the sets of form $f^{-1}(y), y \in Y$ are Baire spaces. If any one of the following conditions holds, then $X$ is a Baire space.

(i) $f$ is weakly-open and weakly-continuous.

(ii) $f$ is weakly-open and $\theta$-continuous. 
(iii) $f$ is weakly-open and almost-continuous.

(iv) $f$ is almost open and almost-continuous.

Before proving these theorems, we need the following Lemmas. At first, we shall prove the relations (a) and (b) in Lemma 2.3 which were given by Z. Frolik in $[9$, p.384] without proof in order to use them in the proof of Lemma 2.4 .

LEMMA 2.3. Let $f$ be a mapping of second countable space $X$ onto a space $Y$. Let $F$ be a subset of $X$ and let $\left\{U_{n} \mid \quad n=1,2, \ldots\right\}$ be an open base for $X$. If $M_{n}=\{y \in Y \mid \emptyset \neq$ $\left.f^{-1}(y) \cap U_{n} \subset F\right\}$ and $M(F)$ is the set of all $y \in Y$ for which the interior of $f^{-1}(y) \cap F$ (with respect to $\left.f^{-1}(y)\right)$ is nonempty, then the following properties hold.

(a) $M_{n}=Y-f\left(U_{n} \cap(X-F)\right)$

(b) $M(F)=\bigcup_{n=1}^{\infty} M_{n}$

To prove the part (a) suppose that $y$ belongs to $M_{n}$. Then $f^{-1}(y) \cap U_{n} \subset F$. By taking complements, we have $(X-F) \subset\left(X-f^{-1}(y)\right) \cup\left(X-U_{n}\right)$. From this we obtain $U_{n} \cap(X-F) \subset$ $f^{-1}(Y-\{y\})$. This implies that $f\left(U_{n} \cap(X-F)\right) \subset Y-\{y\}$. Therefore y belongs to complement of $f\left(U_{n} \cap(X-F)\right)$. Hence $M_{n} \subset Y-f\left(U_{n} \cap(X-F)\right)$ For the reverse inclusion, let $\mathrm{y}$ be an arbitrary point in $Y-f\left(U_{n} \cap(X-F)\right)$, then $f^{-1}(y) \subset f^{-1}\left[Y-f\left(U_{n} \cap(X-F)\right)\right]$ and $f^{-1}(y) \subset X-f^{-1}\left[f\left(U_{n} \cap(X-F)\right)\right] \subset X-\left(U_{n} \cap(X-F)\right)$. Consequently, $f^{-1}(y) \subset\left(X-U_{n}\right) \cup F$. This implies that $f^{-1}(y) \cap U_{n} \subset F$. Hence y belongs to $M_{n}$. Thus $Y-f\left(U_{n} \cap(X-F)\right) \subset M_{n}$. This completes the proof of (a).

The proof of the part (b) Suppose that y belongs to $\mathrm{M}(\mathrm{F})$. Then $\operatorname{int}_{f^{-1}(y)}\left(f^{-1}(y) \cap F\right) \neq \emptyset$ by the definition of $\mathrm{M}(\mathrm{F})$. Since $\left\{U_{n} \mid n=1,2, \ldots\right\}$ is an open base for $\mathrm{X}$ then there exist a positive integer $n_{0}$ such that $\emptyset \neq f^{-1}(y) \cap U_{n_{0}} \subset f^{-1}(y) \cap F$. This shows that y belongs to $M_{n_{0}}$. Consequently this impies that $y \in \bigcup_{n=1}^{\infty} M_{n}$. Hence $M(F) \subset \bigcup_{n=1}^{\infty} M_{n}$. To prove the reverse inclusion, let $\mathrm{y}$ be an arbitrary point in $\bigcup_{n=1}^{\infty} M_{n}$. Then there is a positive integer $n_{0}$ such that $y \in M_{n_{0}}$. Thus $\emptyset \neq f^{-1}(y) \cap U_{n_{0}} \subset F$. by the definition of $M_{n_{0}}$. This means that $\operatorname{int}_{f^{-1}(y)}\left(f^{-1}(y) \cap F\right) \neq \emptyset$. Therefore y belongs to $\mathrm{M}(\mathrm{F})$. Hence $\bigcup_{n=1}^{\infty} M_{n} \subset M(F)$. This completes the proof of (b).

LEMMA 2.4. Let $f$ be a mapping of a second countable space $X$ onto a space $Y$. Let $F$ be a nowhere dense closed subset of $X$. Denote by $M(F)$, the set of all $y \in Y$ for which the interior of $f^{-1}(y) \cap F$ (with respect to $f^{-1}(y)$ ) is nonempty. If any one of the following conditions holds then the set $M(F)$ is a first category subset of $Y$.

(i) $f$ is semi-open and semi-continuous.

(ii) $f$ is semi-open and almost-continuous.

(iii) $f$ is feebly-open and almost-continuous.

PROOF. Suppose that (i) holds and let $\left\{U_{n} \mid \quad n=1,2, \ldots\right\}$ be open base for $X$. Let put $M_{n}=\left\{y \in Y \mid \quad \emptyset \neq f^{-1}(y) \cap U_{n} \subset F\right\}$. Now suppose that $M(F)$ is not of the first category subset of $Y$. Since $M(F)=\bigcup_{n=1}^{\infty} M_{n}$ by Lemma 2.3, then there exists a positive integer $n_{0}$ such 
that $\operatorname{int}\left(\overline{M_{n_{0}}}\right) \neq \emptyset$. On the other hand, since $f$ is semi-open and

$$
M_{n_{0}}=Y-f\left(U_{n_{0}} \cap(X-F)\right)
$$

by part (a) of Lemma 2.3 , then $M_{n_{0}}$ is semi-closed and consequently $\operatorname{int}\left(\overline{M_{n_{0}}}\right) \subset \operatorname{int} M_{n_{0}}$ by Theorem 1.24. Thus int $M_{n_{0}} \neq \emptyset$. By the definition of $M_{n_{0}}$

$$
\emptyset \neq f^{-1}\left(\operatorname{int} M_{n_{0}}\right) \cap U_{n_{0}} \subset F
$$

Since $f$ is semi-continuous, then $f^{-1}\left(\operatorname{int} M_{n_{0}}\right)$ is semi-open and $f^{-1}\left(\operatorname{int} M_{n_{0}}\right) \cap U_{n_{0}}$ is also semi-open by Theorem 1.4. Hence there is a nonempty open subset $O$ of $X$ such that

$$
O \subset f^{-1}\left(\operatorname{int} M_{n_{0}}\right) \cap U_{n_{0}} \subset \bar{O} .
$$

However, this would contradict $F$ being nowhere dense in $X$.

Now suppose that (ii) holds and $M(F)$ is not a first category subset of $Y$. Since the mapping $f$ as in the case of (i) is semi-open, then by repeating the same operations, we can obtain the following result,

$$
\emptyset \neq f^{-1}\left(\operatorname{int} M_{n_{0}}\right) \cap U_{n_{0}} \subset F
$$

since $\operatorname{int} \overline{M_{n_{0}}}$ is regular open and $f$ is almost-continuous, then $f^{-1}\left(\operatorname{int} \overline{M_{n_{0}}}\right.$ ) is open by Theorem 1.14 and consequently $F$ is not nowhere dense subset in $X$. This is a contradiction.

The proof for the case (iii) is a direct consequence of the case (ii) of this lemma by the case (iii) of Theorem 1.18.

The following Theorem now makes it easy to prove Theorem 2.1. and Theorem 2.2.

THEOREM 2.5. Let $f$ be a mapping of a second countable space $X$ onto a second category space $Y$ and suppose the sets of the form $f^{-1}(y), y \in Y$ are second category spaces. If any one of the following conditions holds, then $X$ is a second category space.

(i) $f$ is semi-open and semi-continuous.

(ii) $f$ is semi-open and almost-continuous.

(iii) $f$ is feebly-open and almost-continuous.

This theorem is proved by using the same method as in $\left[9\right.$, Theorem $\left.2^{\prime}, \mathrm{p} .384\right]$. Therefore the proof is omitted.

COROLLARY 2.6. Let $f$ be a mapping of a metrizable separable space $X$ onto a second category space $\mathrm{Y}$ and suppose the sets of the form $f^{-1}(y), y \in Y$ are second category spaces. If any one of the following conditions hold, then $\mathrm{X}$ is a second category space.

(i) $f$ is weakly-open and weakly-continuous

(ii) $\mathrm{f}$ is weakly-open and $\theta$-continuous

(iii) $f$ is weakly-open and almost-continuous

(iv) $f$ is almost-open and almost-continuous

PROOF. Suppose that (i) holds. By the metrizability and separability (actually regularity and second countability) of $X$, the proof follows from Theorem 1.23, Theorem 1.12 and the case 
(ii) of Theorem 2.5. It is clear that both the case (ii) and the case (iii) imply the case (i) by Remark 1.9. Finally the proof of the case (iv) follows from case (iii) of this corollary by Remark 1.20 .

COROLLARY 2.7. Let $\mathrm{f}$ be a mapping of a second countable space $X$ onto an almostregular second category space $Y$ and suppose the sets of the form $f^{-1}(y), y \in Y$ are second category spaces. If any one of the following conditions hold, then $\mathrm{X}$ is a second category space.

(i) $f$ is feebly-open and weakly-continuous.

(ii) $\mathrm{f}$ is feebly-open and $\theta$-continuous.

PROOF. Suppose that (i) holds. Since $Y$ is almost-regular, $f$ is feebly-open and almostcontinuous by Theorem 1.11. Consequently $X$ is a second category space by the case (iii) of Theorem 2.5. The proof for the case (ii) follows from Remark1.9 and the case (i) of this corollary.

PROOF OF THEOREM 2.1. In order to prove that $X$ is a Baire space, it is enough to show that every nonempty open subset of $X$ is of the second category (also called nonmeager) relative to $X$. Let $G$ be an arbitrary nonempty open subset of $X$. Since $f$ is semi-open, then $f(G)$ is a semi-open subset in $Y$. Therefore, there exists a nonempty open subset $O$ of $Y$, such that $O \subset f(G) \subset \bar{O}$. Thus $f(G)$ is a Baire subspace of $Y$ by Theorem 1.25 and Theorem 1.26. Consequently $f(G)$ is a second category subspace in $Y$. On the other hand, the mapping $f_{/ G}: G \longrightarrow f(G)$ defined by $f_{/ G}(x)=f(x)$ for all $x \in G$, is semi-open and semi-continuous by Theorem 1.6 and Theorem 1.7. Furthermore since the sets form $f^{-1}(y)$ are Baire spaces and $\left(f_{/ G}\right)^{-1}(y)=G \cap f^{-1}(y)$, then the sets form $\left(f_{/ G}\right)^{-1}(y)$ are open Baire subspaces of $f^{-1}(y)$. by Theorem 1.25. Consequently the sets of form $\left(f_{/ G}\right)^{-1}(y)$ are also Baire subspaces of $G$ by Remark 1.28. Hence the sets of form $\left(f_{/ G}\right)^{-1}(y)$ are second category subspaces of G. Therefore $G$ is a second category subspace in $X$ by the case(i) of Theorem 2.5. Consequently $G$ is a second category subset relative to $X$ by Theorem 1.27 .

PROOF OF THEOREM 2.2. Suppose that (i) holds and let $G$ be an arbitrary nonempty open subset of $X$. Since $X$ is metrizable, $f$ is open and almost-continuous by Theorem 1.23 and Theorem 1.12. Hence $f(G)$ is an open Baire subspace of $Y$ by Theorem 1.25. Consequently $f(G)$ is a second category subspace of $Y$. Moreover the mapping $f_{/ G}: G \longrightarrow f(G)$ is also open and almost continuous by Theorem 1.13. On the other hand, as in the proof of Theorem 2.1, by repeating the same operations, it can be shown that the sets of form $\left(f_{/ G}\right)^{-1}(y)$ are second category subspaces of G. Consequently $G$ is a subspace of the second category in $X$ by the case (ii) of Theorem 2.5. This shows that $G$ is a second category subset relative to $X$ by Theorem 1.27 .

It is clear that both the case (ii) and the case (iii) imply the case (i) by Remark 1.9. Finally the proof of the case (iv) follows from case (iii) of this theorem by Remark 1.20.

ACKNOWLEDGMENT. The author express greatful thanks to referees for some valuable remarks. 


\section{REFERENCES}

[1] AARTS, J. M. and LUTZER, D.J., Completeness properties designed recognizing Baire spaces, Dissertationes math. 116(1974) 1-48.

[2] BISWAS, N. , On some mappings in topological spaces, Bull. Calcutta. Math. Soc. 61(1969) 127-135.

[3] - On characterizations of semi-continuous function, Atti. Accad. Naz. Lincei. Rend. Cl. Sci. Fis. Math. Natur. (8) 48(1970) 399-402.

[4] BOURBAKI, N. , Elements of Mathematics General Topology, Volume 2 (Hermann, Paris, 1966).

[5] CHOQUET, G., Lectures on analysis. Vol I. Benjamin, New York, 1969

[6] DIEUDONNE, J. , Foundations of modern analysis, Academic Press, New York, 1969

[7] CROSLey, S. G. and HILdebrand, S. K. , Semi-closure, The Texas Journal of Science, Vol. XXII. No 283(1971) 99-112.

[8] FOMIN, S., Extensions of topological spaces, Ann. math. 44(1943) 471-480.

[9] FROLIK, Z. , Remarks concerning the invariance of Baire spaces under mappings, Czech. Math. J. 11(86) (1961) 381-384.

[10] — Baire spaces and some Generalizations of complete metric space, Czech. Math. J. $11(86)(1961)$ 237-248.

[11] HAWORTH, R. C. and MCCOY, R.A., Baire spaces, Dissertationes. Math. 141(1977) 1-73.

[12] KIM, H.O., Notes on C-compact Spaces and Functionally Compact Spaces, Kyungpook Math.J., Vol. 10(1970), 75-80.

[13] KROM, M.R., Cartesian product of metric Baire spaces, Proc. Amer. Math. Soc. 42(1974) 588-594.

[14] LEVINE, N., A decomposition of continuity in topological spaces, Amer. Math. Monthly. $68(1961)$ 44-46.

[15] - semi-open sets and semi-continuity in topological spaces, Amer. Math. Monthly. $70(1963) 36-41$.

[16] LONG, P.E. and HERRINGTON, L. L. , Properties of almost-continuous functions, Bollettino. U. M. I. (4) 10(1974) 336-342. 
[17] NOIRI, T., Between continuity and weak-continuity, Bollettino U.M.I (4)9(1974),647-654.

[18] OXTOBY, J.C., Cartesian product of Baire spaces, Fund. Math. 49(1960/61), 157-166.

[19] ROSE, D.A., Weak opennes and almost opennes, Internat. J. Math and Math. Sci.. $7(1984)$ no.1,35-40.

[20] SIKORKSKI, R., On the cartesian product of metric spaces, Fund.Math.34(1947), 288292.

[21] SINGAL, M.K. and SINGAL, A.R. , almost-continuous mappings, Yokohoma. Math. J. 16(1968) 63-73.

[22] SINGAL, M. K. and ARYA S. P., On almost regular spaces, Glasnik. Mat. Seri. III 4(24) (1969) 89-99.

[23] STEEN, L.A. and SEEBACH, J.A.,Jr., Counterexamples in toloogj, Holt, Rinehart and Winston, New York, 1970.

[24] NEUBRUNN, T., A note on mappings of Baire space, Math. Slovaca 27(1977) 173-176. 


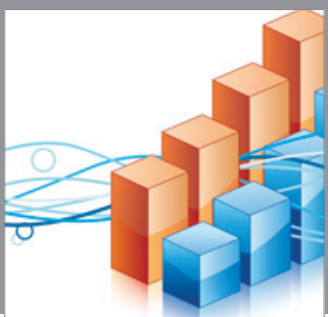

Advances in

Operations Research

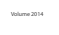

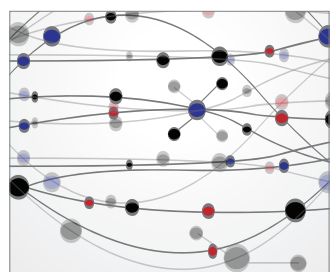

\section{The Scientific} World Journal
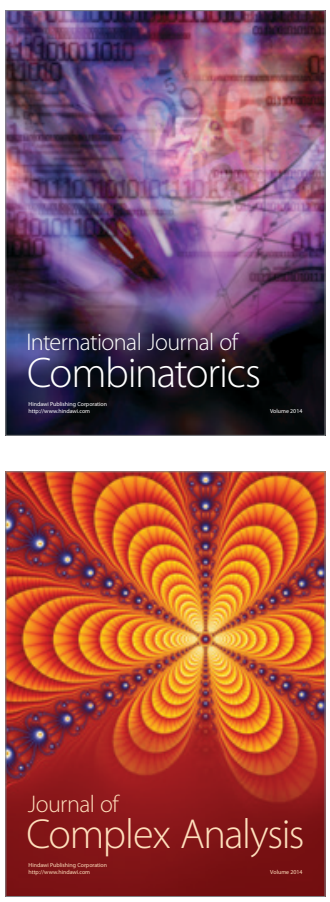

International Journal of

Mathematics and

Mathematical

Sciences
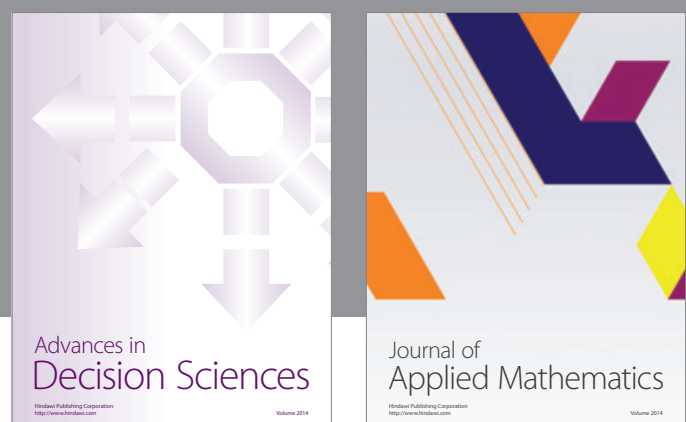

Journal of

Applied Mathematics
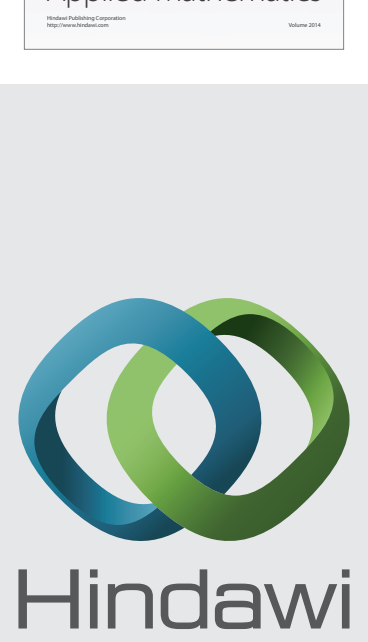

Submit your manuscripts at http://www.hindawi.com
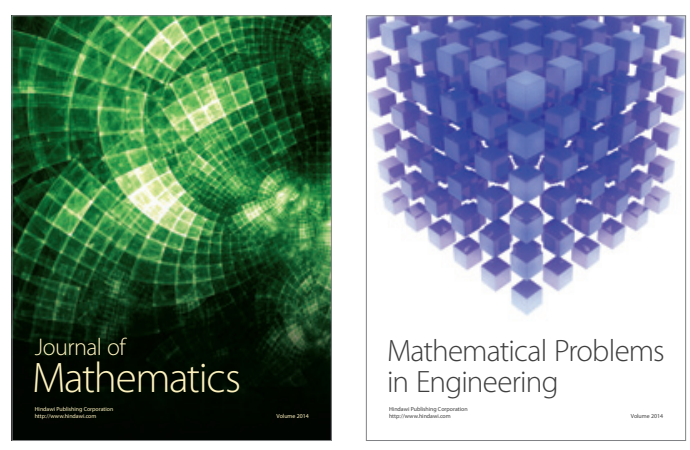

Mathematical Problems in Engineering
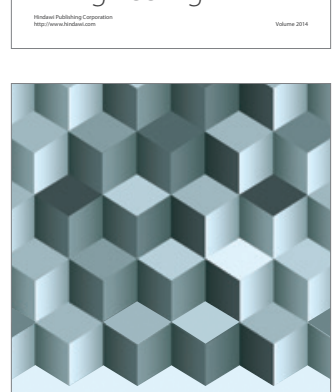

Journal of

Function Spaces
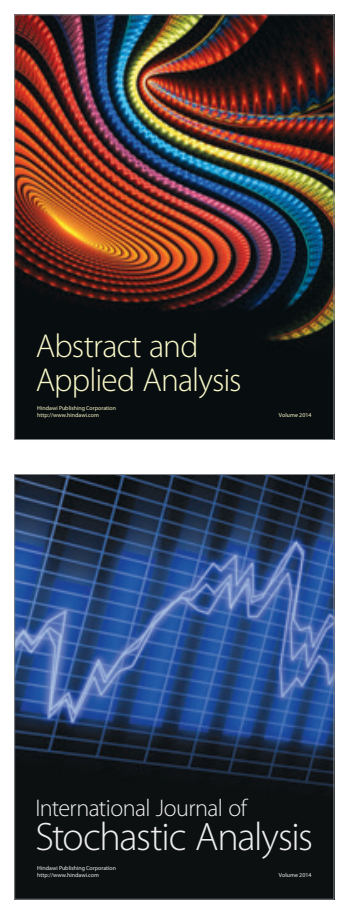

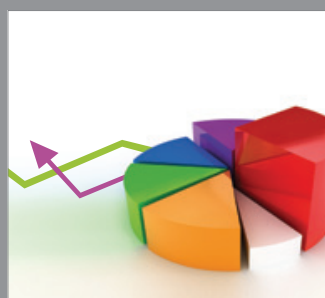

ournal of

Probability and Statistics

Promensencen
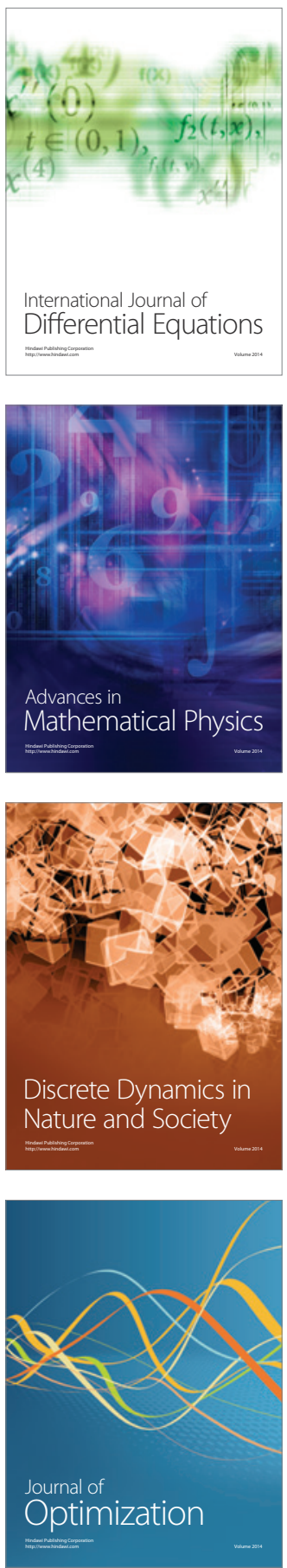